\title{
High Directivity of Microstrip Patch Antenna using Metamaterial
}

\author{
Muhammad Waqas ${ }^{1}$, Shahid Bashir ${ }^{2}$, Muhammad Junaid Khan ${ }^{3}$, \\ Zeeshan Akbar ${ }^{4}$ \\ ${ }^{l}$ Department of Electrical Engineering, Sarhad University of Science and Information Technology, Peshawar, \\ Pakistan \\ ${ }^{2}$ Department of Electrical Engineering, University of Engineering and Technology, Peshawar, Pakistan \\ ${ }^{3}$ Department of Communications Engineering, University of Oulu, Finland, \\ ${ }^{4}$ Department of Electrical Engineering, Sarhad University of Science and Information Technology, Peshawar,
} Pakistan

\begin{abstract}
In this paper, a different Double Negative (DNG) metamaterial is demonstrated to upgrade the performance of Microstrip Patch Antenna (MPA). The scattering parameters are utilized to plan and corroborate the DNG structure. The proposed DNG is then employed as a superstrate of MPA which reduces the surface waves and edge diffracted waves of an antenna and therefore reduces its losses. To analyze the performance a conventional MPA without DNG is also simulated. The outcomes indicate that DNG based MPA has enhanced performance as far as directivity, gain and radiation efficiency of antenna. The directivity is improved from 6.878dBi to 7.184dBi. The effectiveness of an antenna is increased from $72 \%$ to $80.42 \%$. Gain of antenna is also enhanced from $5.454 \mathrm{~dB}$ to $6.237 \mathrm{~dB}$. The proposed circular SRR based DNG MPA could be a great competitor for application in wireless communication.
\end{abstract}

Keywords: Double negative metamaterial, rectangular microstrip patch antenna, negative permittivity and permeability, high directivity

\section{Introduction}

A conventional MPA has various provisions in microwave applications however because of its low gain, directivity and efficiency it has restricted applications. A metamaterial is proposed to enhance these essential parameters of MPA. Metamaterials are engineered structures that show certain electromagnetic characteristics which are not discovered in nature. These structures are categorized by negative values for both permittivity and permeability [1]. These materials are likewise considered Double negative materials (DNM) or left handed materials (LHM) [2]-[5]. The idea of metamaterials presence is initially anticipated by Russian Physicist Victor Veselago in 1968 [6] however the exploratory confirmation did not happen until several decades. Diverse methods and structures of DNG metamaterial are proposed to improve the characteristics of MPA [7]-[14]. These DNG material structures are basically used as a superstrate for ordinary MPA [15]-[18] In this work another sort of DNG metamaterial is proposed, which is comprising of circular split ring resonators (SRR) on one side of the dielectric medium and three circular shape wires on the other side of a medium as a superstrate.

An MPA has various benefits because of its minimal effort, lightweight, simple to create and reconcile with different electronic circuitry [19], [20]. So as to enhance these parameters this paper proposes another DNG metamaterial which is used as a superstrate at a height of $3.36 \mathrm{~mm}$ starting from ground plane. The outcomes demonstrated that the directivity, gain, return loss have been effectively enhanced. The directivity is improved from $6.878 \mathrm{~dB}$ to $7.184 \mathrm{~dB}$. Gain and radiation proficiency of an antenna is improved by $0.783 \mathrm{~dB}$ and $8.42 \%$ respectively while return loss is reduced to $-28.17 \mathrm{~dB}$ from $-16.55 \mathrm{~dB}$. All parameters an antenna has been considerably improved due to DNG metamaterial as superstrate of customary MPA.

Simulations are completed utilizing 3D EM Simulator Computer Simulation Technology Microwave Studio (CST-MWS) [21]. MATLAB is used for the verification of Double Negative properties of the proposed DNG structure.

\section{Methodology}

In the first stage a conventional rectangular microstrip patch antenna (RMPA) is designed as shown in Fig.1. 


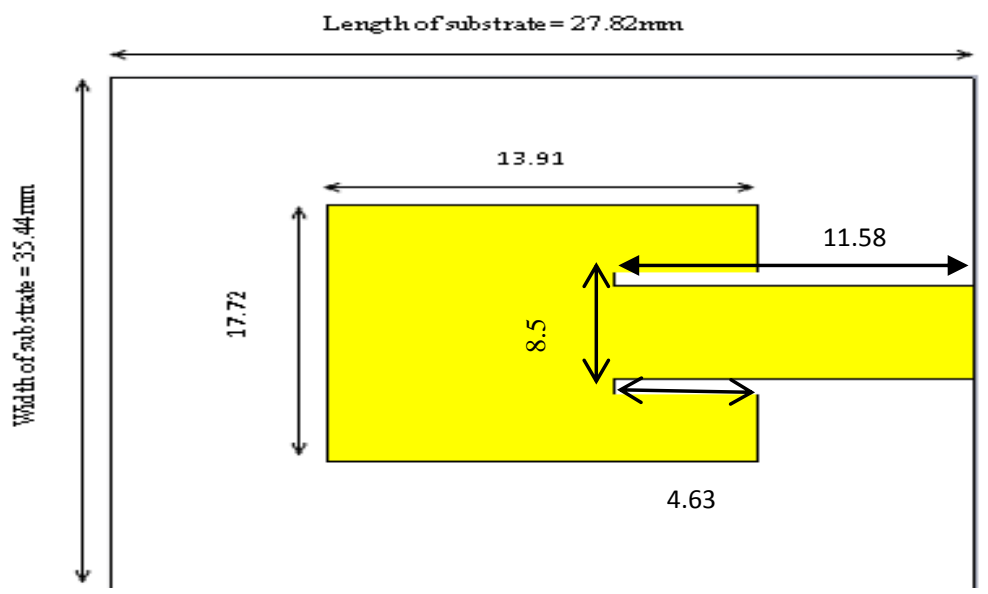

Fig.1: Conventional RMPA (All dimensions are in $\mathrm{mm}$ )

Parameters of RMPA are calculated from the given equation in [22].

Calculation of width

$W=\frac{1}{2 f_{r} \sqrt{\varepsilon_{0} \mu_{0}}} \sqrt{\frac{2}{\varepsilon_{r}+1}}=\frac{C}{2 f_{r}} \sqrt{\frac{2}{\varepsilon_{r}+1}}$

Where,

$\mathrm{C}=$ Speed of light

$\varepsilon_{r}=$ Dielectric constant of substrate

Effective dielectric constant is calculated from,

$\varepsilon_{e f f}=\frac{\varepsilon_{r}+1}{2}+\frac{\varepsilon_{r}-1}{2}\left(\frac{1}{\sqrt{1+\frac{12 h}{W}}}\right)$

The actual length of patch (L),

$L=L_{e f f}-2 \Delta L$

Where,

$L_{e f f}=\frac{C}{2 f_{r} \sqrt{\varepsilon_{e f f}}}$

Calculation of length extension,

$\frac{\Delta L}{h}=0.412 \frac{\left(\varepsilon_{e f f}+0.3\right)\left(\frac{W}{h}+0.264\right)}{\left(\varepsilon_{e f f}-0.258\right)\left(\frac{W}{h}+0.8\right)}$

The calculated parameters of RMPA are given in Table I.

Table I: Parameters of RMPA

\begin{tabular}{|l|l|l|}
\hline Parameters & Dimensions & Units \\
\hline Dielectric constant $\left(\varepsilon_{\mathrm{r}}\right)(\mathrm{FR} 4)$ & & \\
\hline Thickness $(\mathrm{H})$ & 4.3 & - \\
\hline Operating frequency $\left(\mathrm{f}_{\mathrm{r}}\right)$ & 1.0 & $\mathrm{~mm}$ \\
\hline Length of Patch $(\mathrm{L})$ & 5.2 & $\mathrm{GHz}$ \\
\hline Width of patch $(\mathrm{W})$ & 13.91 & $\mathrm{~mm}$ \\
\hline Cut width & 17.72 & $\mathrm{~mm}$ \\
\hline
\end{tabular}




\begin{tabular}{|l|l|l|}
\hline Cut depth & 4.63 & $\mathrm{~mm}$ \\
\hline Length of microstrip & 11.58 & $\mathrm{~mm}$ \\
\hline Width of microstrip & 6.5 & $\mathrm{~mm}$ \\
\hline Length of substrate \& ground & 27.82 & $\mathrm{~mm}$ \\
\hline Width of substrate \& ground & 35.44 & $\mathrm{~mm}$ \\
\hline
\end{tabular}

The antenna is modeled and simulated in CST MWS. The S11 graph shows that antenna resonate at 5.2 $\mathrm{GHz}$ as shown in Fig.2.

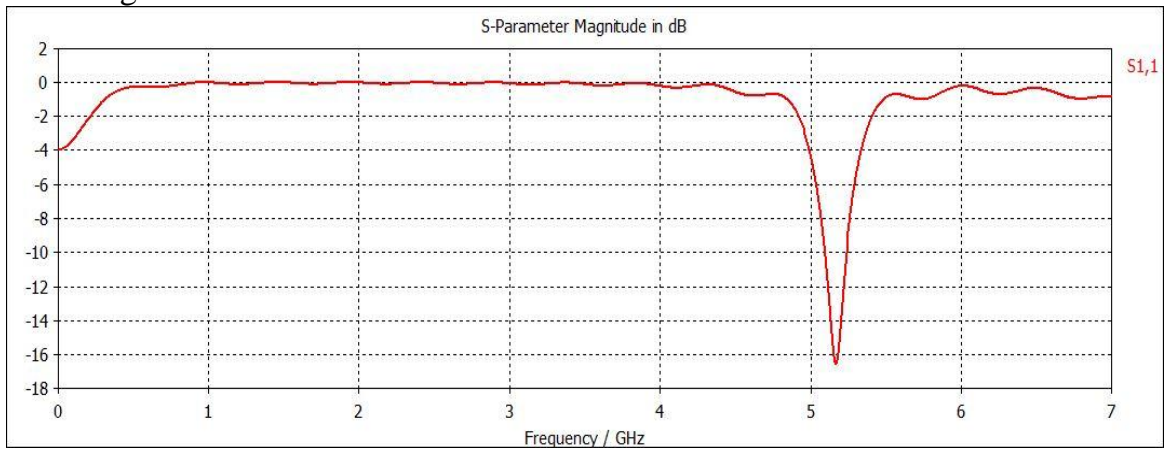

Fig.2: Impedance matching of RMPA

To extract scattering parameters S11 and S21, the proposed design DNG structure is placed between two waveguide ports in CST MWS as shown in Fig.3.

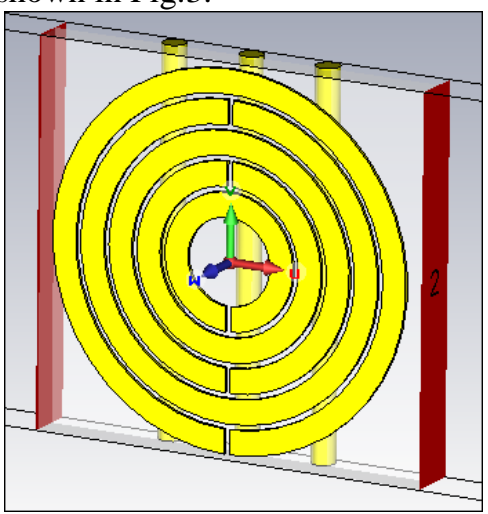

Fig.3: DNG structure between two waveguide ports

The S11 and S21 parameters are then operated to Matlab for the verification of negative permittivity and permeability of DNG structure by direct retrivel technique [23]. The permitivity and permeability parameters are negative in the given frequncy band as indicated in Fig.4 and Fig.5 respectively. The equations used in direct retrieval method to find permittivity and permeability is given as:

$$
\begin{aligned}
& \varepsilon=n / z \\
& \mu=n z \\
& n=\frac{1}{k d} \cos ^{-1}\left[\frac{1}{2 S_{21}}\left(1-S_{11}^{2}+S_{21}^{2}\right)\right] \\
& z=\sqrt{\frac{\left(1+S_{11}\right)^{2}-S_{21}^{2}}{\left(1-S_{11}\right)^{2}-S_{21}^{2}}}
\end{aligned}
$$

The dimension of DNG structure is given in Table II.

Table II: Dimensions of DNG structure

\begin{tabular}{|c|c|c|}
\hline Parameters of DNG & Values & Unit \\
\hline Length of each wire & 14.2 & $\mathrm{~mm}$ \\
\hline Radius of each wire & 0.45 & $\mathrm{~mm}$ \\
\hline Inner and outer radius of ring 1 & $2.5 \& 1.6$ & $\mathrm{~mm}$ \\
\hline Inner and outer radius of ring 2 & $3.6 \& 2.7$ & $\mathrm{~mm}$ \\
\hline Inner and outer radius of ring 3 & $4.7 \& 3.8$ & $\mathrm{~mm}$ \\
\hline Inner and outer radius of ring 4 & $5.8 \& 4.9$ & $\mathrm{~mm}$ \\
\hline Inner and outer radius of ring 5 & $6.9 \& 6.0$ & $\mathrm{~mm}$ \\
\hline Dielectric of substrate & 4.3 & $\mathrm{~mm}$ \\
\hline Thickness of substrate (FR4) & 1 & $\mathrm{~mm}$ \\
\hline
\end{tabular}




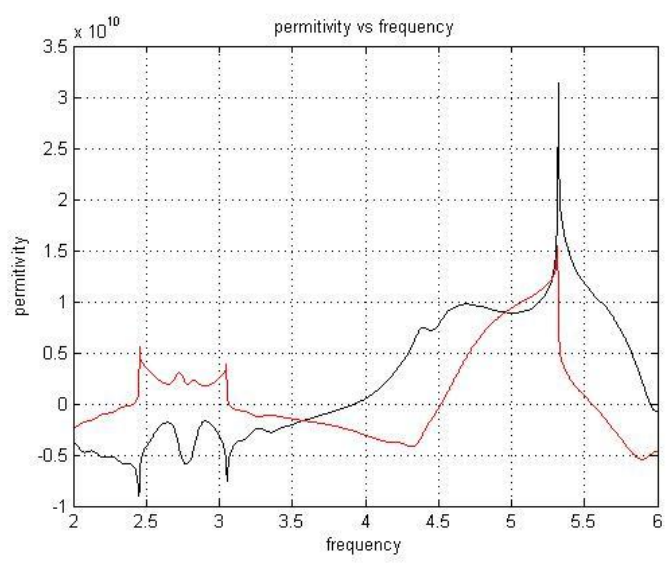

Fig.6: Proposed DNG permittivity vs frequency

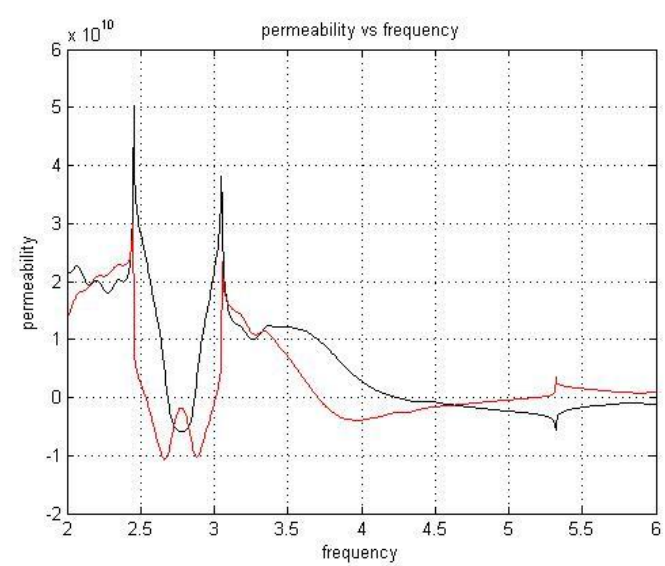

Fig.7: Proposed DNG permeability vs frequency

DNG metamaterial structure is placed as superstrate on RMPA shown in Fig. 8 at height of $3.36 \mathrm{~mm}$ from the ground plane in the final stage of work and compared the results of DNG based antenna with conventional antenna.

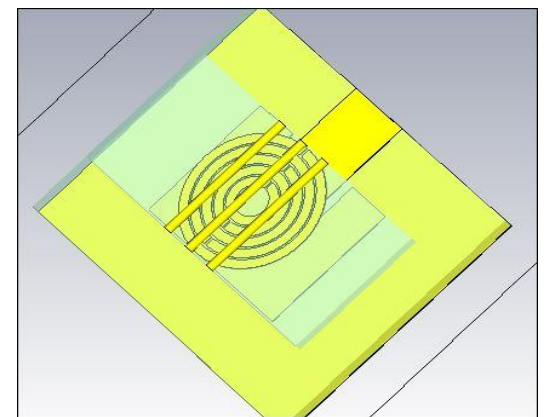

Fig.8: Microstrip patch antenna with DNG superstrate

\section{Results and Discussion}

Several parameters like gain, directivity, radiation efficiency and return losses of antenna with transmission line are simulated and compared between conventional MPA and DNG based MPA is discussed below.

\subsection{Impedance matching}

S11-Parameter of DNG based antenna is shown in Fig.9. It is observed that resonant frequency remain unchanged while impedance matching improved for DNG based MPA. 


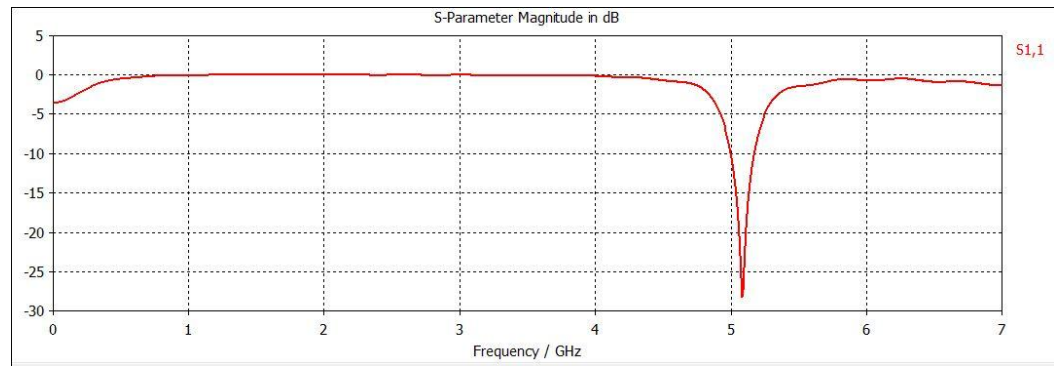

Fig.9: Impedance matching of DNG based RMPA

\subsection{Farfield Pattern}

Directivity and radiation efficiency of DNG based antenna have been examined and compared for conventional MPA and DNG based MPA. From given Fig.10 and Fig.11 it is evident that directivity of MPA is enhanced from $6.878 \mathrm{dbi}$ to $7.184 \mathrm{dbi}$. The improved radiation efficiency due to DNG based MPA is increased by $8.42 \%$.

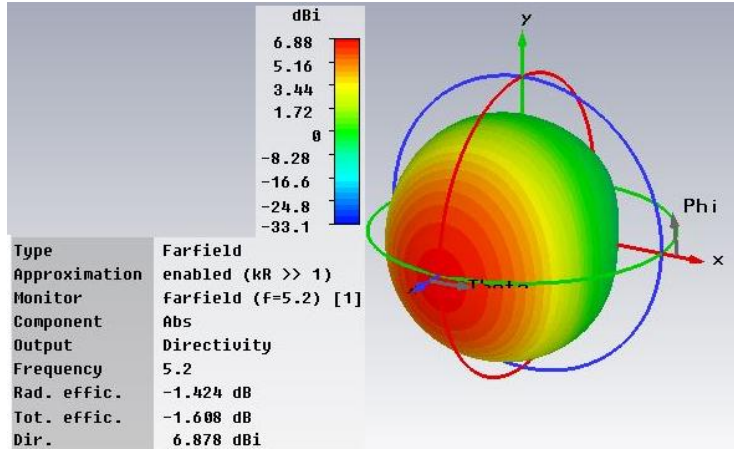

Fig.10: Directivity of RMPA

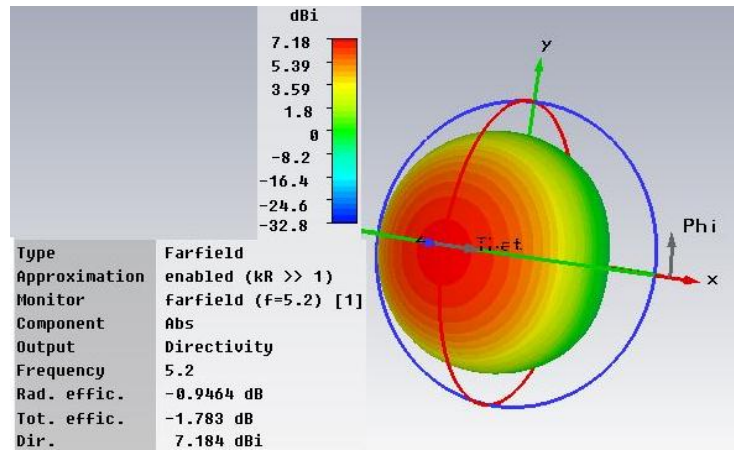

Fig.11: Directivity of DNG based MPA

The gain of DNG based MPA is also improved form $5.454 \mathrm{~dB}$ to $6.237 \mathrm{~dB}$ as shown in Fig. 12 and Fig.13 respectively.

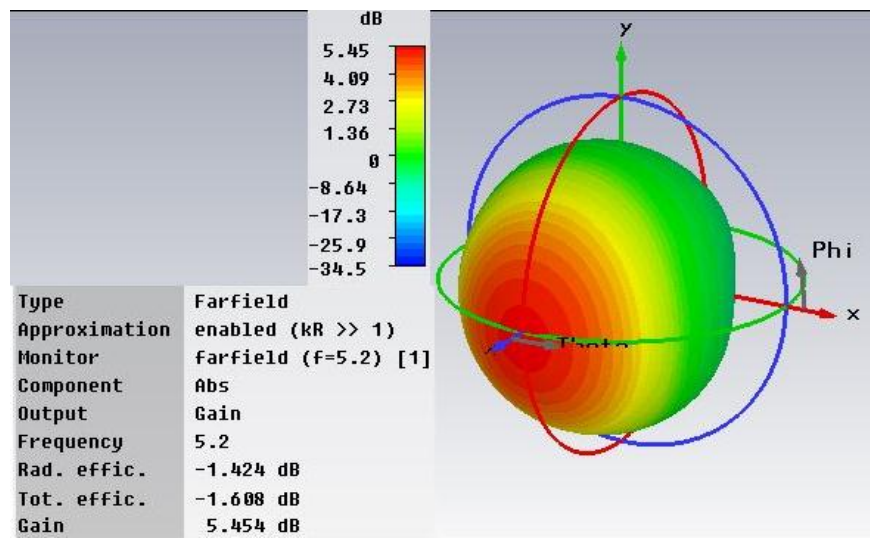

Fig12: Gain of RMPA 


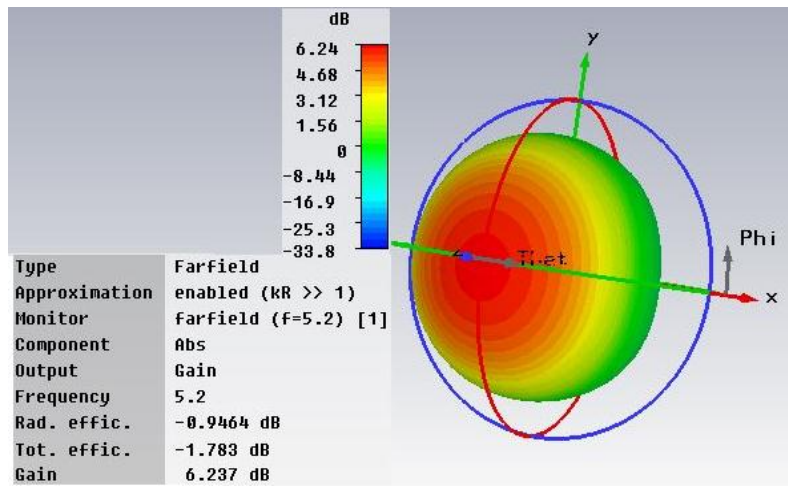

Fig.13: Gain of DNG based MPA

\subsection{Radiation Efficiency of Antenna}

Radiation efficiency of conventional MPA is $-1.424 \mathrm{~dB}(72 \%)$ but when DNG superstrate is taken into account its radiation efficiency is increased to $-0.9464 \mathrm{~dB}$ which is $80.42 \%$.

\section{Conclusion}

The DNG based MPA usefulness has been completed. The DNG material reduces losses of an antenna which improved its directivity, gain and radiation efficiency. The directivity and gain of DNG based MPA is improved from $5.454 \mathrm{~dB}$ to $6.237 \mathrm{~dB}$ and from $6.878 \mathrm{~dB}$ to $7.18 \mathrm{~dB}$ respectively. The return loss of an antenna is reduced by $11.62 \mathrm{~dB}$. Consequently its radiation efficiency is increased from $72 \%$ to $80.42 \%$. The DNG based MPA can be used in advance wireless communication. The overall improvement in performance between conventional MPA and DNG based MPA are summarized in Table III.

Table III: Comparison between Conventional MPA and DNG based MPA

\begin{tabular}{|c|c|c|c|}
\hline S.No & Parameters & Conventional RMPA & RMPA with superstrate \\
\hline 1 & Frequency & $5.2 \mathrm{GHz}$ & $5.2 \mathrm{GHz}$ \\
\hline 2 & Directivity & $6.878 \mathrm{~dB}$ & $7.18 \mathrm{~dB}$ \\
\hline 3 & Radiation efficiency & $-1.424 \mathrm{~dB}(62.28 \%)$ & $-0.9464 \mathrm{~dB}(86.73 \%)$ \\
\hline 4 & Gain & $5.454 \mathrm{~dB}$ & $6.237 \mathrm{~dB}$ \\
\hline 5 & $\begin{array}{c}\text { Scattering Parameters } \\
\text { (S11) }\end{array}$ & $-16.55 \mathrm{~dB}$ & $-28.17 \mathrm{~dB}$ \\
\hline
\end{tabular}

\section{References}

[1] L. Angeles, “Compsite Right/Left -Handed Transmission Line Metamaterial," IEEE Microwave Magzine, pp. 34-50, September 2004.

[2] V. G. Veselago, "The Electrodynamics of Substances with Simultaneously Negative values of $\varepsilon$ and $\mu$, Soviet Phys Usp 10 (1968), 509-514.

[3] A. Mallik, S. Kundu, and O. Goni, "Design of a novel Two-rectangular U-Shaped Double Negative Metamaterial," IEEE no. 978-14799-0400-6/2013

[4] J. Franklin, J. Biddle, Bohdan Balko, "Double Negative Materials ( DNM ) Phenomena and Applications," Institute of Defense Analysis central research Program, July 2009.

[5] R. Marques, F. Martin, M. Sorolla, "Metamaterial with negative paramters, theory, design and microwave applications," John Wiley \& Sons, Inc. 2008.

[6] M. F. Khan, "Tunable metamaterials," PhD. thesis, Ghulam Ishaq Khan Institute of Engineering Sciences and Technology, Topi, Swabi, Khyber Pakhtunkhwa, Pakistan. May 2011.

[7] Bimal Garg, Nitin Agrawal, Vijay Sharma, Ankita Tomar, Prashant Dubey, "Rectangular microstrip patch antenna with pentagonal rings shaped metamaterial," Int. conf. on Communication Systems and Network Technologies, no. 5, pp. 41-45, 2012.

[8] P. K. Singhal, B. Garg, and N. Agrawal, "Techniques in a high gain rectangular microstrip patch antenna using different c patterns metamaterial design in L-band,” Int. Scienctific Publication and Consulting Service, vol. 2012, 2012. Available:

http://www.ispacs.com/acte

[9] P. K. Singhal and B. Garg, "Design and characterization of compact microstrip patch antenna using split ring shaped metamaterial structure,” Int. Journal of Electrical and Computer Engineering, vol. 2, no. 5, pp. 655-662, 2012.

[10] G. He, R. Wu, Y. Poo, and P. Chen, "Magnetically tunable double negative material composed of ferrite dielectric and metallic mesh," Journal of Applied Physics, vol. 093522, Published, May 2012.

[11] A. M. Ali and J. Venkataraman, "Gain enhancement of patch antenna using double negative superstrate realized by a high dielectric with triangular lattice of holes," 2009 IEEE Antennas Propag. Soc. Int. Symp., no. 1, pp. 1-4, June 2009.

[12] B. Y. Dong and T. Itoh, "Metamaterial based antennas," vol. 100, no. 7, pp. 2271-2285, March 19, 2012.

[13] H. Attia, L. Yousefi, M. M. Bait-suwailam, M. S. Boybay, and O. M. Ramahi, "Enhanced gain microstrip antenna using engineered magnetic superstrates," IEEE Antennas and Wireless Prog. Lett. vol. 8, pp. 1198-1201, 2009.

[14] B. Garg, R. D. Verma, and A. Samadhiya, "Design of rectangular microstrip patch antenna incorporated with innovative metamaterial structure for dual band operation and amelioration in patch antenna parameters with negative $\mu$ and $\varepsilon$," Int. Journal of Engg. and Tech. vol. 1, no. 3, pp. 205-216, 2012.

[15] D. Jin, B. Li, and J. Hong, "Gain improvement of a microstrip patch antenna using metamaterial superstrate with the zero refractive index," IEEE Trans., pp. 10-12, 2012. 


\section{High Directivity of Microstrip Patch Antenna using Metamaterial}

[16] R. Mittra, Y. Li, and K. Yoo, “A comparative study of directivity enhancement of microstrip patch antennas with using three different superstrate," Microwave and Optical Technology Lett. vol. 52, no. 2, pp. 327-331, 2010.

[17] P. Baccarelli, P. Burghignoli, G. Lovat and S. Paulotto, "Surface-wave suppression in a double-negative metamaterial grounded slab," IEEE Antennas And Wireless Prop. Lett., vol. 2, pp. 269-272, 2003.

[18] B. Garg, A. Samadhiya, and R. D. Verma, "Analysis and design of microstrip patch antenna loaded with innovative metamaterial structure," Research Journal of Physics and App. Science, vol. 1, no. August, pp. 13-19, August. 2012.

[19] D. R. Jackson, "Overview of microstrip antennas", Department of Electrical Communication Engineering, University of Houston.

[20] C. A. Balanis, Antenna theory analysis and design, $3^{\text {rd }}$ ed., John Wiley \& Sons, INC., New York, 1997, pp. 811-852.

[21] B. Garg, "Analysis and design of left handed metamaterial to ameliorate the bandwidth and return loss using CST," Current Research in Engg. Science and Tech.Journal, vol. 01, no. 03, pp. 73-79, 2013.

[22] B. D. Orban and G. J. K. Moernaut, "The Basics of Patch Antennas." Available: www.orbanmicrowave.com

[23] D. R. Smith, D. C. Vier, and C. M. Soukoulis, "Electromagnetic parameter retrieval from inhomogeneous metamaterials," The American Physical Society, pp. 1-11, March 22, 2005. 